\title{
The Comprehensive Health Center
}

\author{
MILTON TERRIS, M.D., M.P.H.
}

$I^{1}$ $\mathrm{N}$ ANY given period, the theory of public health is realized not only in specific health services and forms of administration but in facilities as well. Theoretical concepts are translated into brick, stone, and concrete. With the passage of time, ideas change in response to altered circumstances, but the buildings persist as monuments to dead doctrines.

When public health was concerned primarily with the prevention of communicable diseases, the dichotomy of preventive and therapeutic medicine was made a cardinal principle in the United States. Public health was defined as the community organization of preventive health services, and its activities were housed in community or district health centers which were, with few exceptions, separate and apart from the therapeutic facilities of the community.

Today when public health is concerned with all disease, whether communicable or not, the isolation of preventive from therapeutic medicine is no longer accepted. Prevention is considered applicable not only to disease occurrence but also to the progress of disease and disability, and it is recognized that therapeutic services often fulfill preventive functions. Public health is now defined as the community organization of all health services, whether concerned with prevention, therapy, or rehabilitation.

\section{Origins}

The redefinition of public health made it necessary to revise traditional concepts of the health center. In the United States, the first major proposal for a new type of health center was made in 1919 by Dr. Hermann Biggs, then commissioner of health of New York State. $\mathrm{He}$ recommended legislation providing State aid for local health centers which would include hospitals as "essential parts of the centre"; clinics for outpatients, both existing public health clinics and medical, surgical, and diagnostic clinics; clinical, bacteriological, and chemical laboratories; district health service, with a district health officer and deputy health officers; public health nursing service; school medical inspection and treatment services; and "headquarters for all health, medical, nursing, and other public welfare activities of the district which wish to utilize the centre" (1). On administration, Biggs proposed that "The District Health Officer may be the superintendent of the hospital and general director of health of the district and of the hospital and medical activities connected therewith."

The Sage-Machold Bill which embodied Biggs' recommendations was introduced without success in the State Legislature in 1920. It had impressive endorsement from national public health leaders, including Theobald Smith, Milton J. Rosenau, C.-E.A. Winslow, and Surgeon General Hugh Cumming. Dr. Charles V. Chapin wrote in support of the bill :

"I have long been of the opinion that the care of the sick should be closely correlated with the more strictly preventive work which is usually considered by the public as the sole function of the health department. Two exceptionally good features of the proposed bill are that it contemplates and provides for such correlation and secondly that it brings out the desirability of the association of outpatient clinic work with hospitals whenever possible. While isolated clinics for certain diseases and in cer-

Dr. Terris is head of the chronic disease unit, division of epidemiology, Public Health Research Institute of the City of New York. 
tain places are doubtless necessary, I believe that it is far better when possible to connect such work as closely as possible with hospitals providing for bed treatment" (1).

Biggs' concept of a comprehensive health center combining community preventive and therapeutic health services, with hospitals as "essential parts of the centre," was not revived for a quarter of a century. In the meantime, however, a somewhat similar proposal, but oriented to group practice and group payment, was made as a primary recommendation of the Committee on the Costs of Medical Care in 1932.

"Medical service, both preventive and therapeutic, should be furnished largely by organized groups of physicians, dentists, nurses, pharmacists, and other associated personnel. Such groups should be organized, preferably around a hospital, for rendering complete home, office, and hospital care" (2).

The committee called for the development of suitable hospitals into "comprehensive community medical centers" and considered it essential that the services of these centers be coordinated with some plan of group payment. In addition to physicians' services, the centers would provide medical social service, home nursing, and housekeeper service, and would make institutional provision, perhaps in nearby suburban or rural territory, for the care of convalescent and chronic patients. The relationship to public health agencies was to be a tenuous one; the headquarters of the health department might "perhaps" be located in the medical center.

Towards the end of World War II, in preparation for the inauguration of a national hospital construction program, Dr. Joseph W. Mountin of the Public Health Service led in urging the establishment of comprehensive health centers through the physical combination of community public health and hospital facilities. "Physical separation between hospitals and health departments," he wrote, "tends to perpetuate the custom under which preventive and curative services, figuratively speaking, operate in different worlds rather than in relation to the same individuals. ... Insofar as circumstances permit, health center facilities should be incorporated in the physi- cal plant of community hospitals" (3). Largely as a result of his efforts, this view became the official policy not only of the Public Health Service, but also of the American Public Health Association and the American Hospital Association. The American Public Health Association recommended that "The desirability of combining hospital facilities with the housing of physicians' offices, clinics, and health departments should be stressed" (4). Later, in a joint statement of policy, the American Hospital Association and the American Public Health Association declared that "Preventive and curative medicine have reached the stage where they are no longer separable, and it is necessary at the present time to bring them together physically and functionally. . . . It is strongly recommended that, wherever circumstances justify and permit, there should be joint housing of hospitals and health departments, and, if possible, the offices of physicians and dentists" (5).

\section{Definition}

The insistent problems of prevention and treatment of chronic disease are now forcing a critical examination of existing health facilities which, with few exceptions, were built to house services concerned with the prevention and treatment of acute illness. The recognition that the chronic diseases are now predominant has been accompanied by a growing dissatisfaction with traditional concepts of both the health center and the hospital. The health center divorced from community treatment facilities is increasingly viewed as an anachronism, while the acute hospital, isolated on the one hand from preventive, ambulatory, and home care services and, on the other, from institutional services for long-term patients, is no longer blindly accepted as an institution capable of meeting the urgent needs of the present day.

Public health services for early detection of the chronic diseases require the closest possible association with hospital and other clinical facilities, while continued supervision to prevent progression and complications of disease is essential for effective treatment of the patient with a chronic illness. Furthermore, the services which such a patient needs vary with 
changes in the status of the disease process; at different times he may require home or office visits from his physician, hospital care for an acute episode, rehabilitation, comprehensive home care, long-term hospital care, or nursing home care. Unless all of these services are fully coordinated, the patient runs serious risk of being lost in the lacunae separating the facilities available for his care.

The comprehensive health center, as envisioned, is designed to meet this pressing need for coordinated health services. It would bring together at one site all the facilities required to provide the preventive, therapeutic, and rehabilitative health services of a local community or district. These include facilities to house the preventive services and clinics of the health department, treatment clinics and offices for physicians and dentists, hospital beds for acute and chronic disease including mental illness, rèhabilitation services, comprehensive home care, and nursing home services.

\section{Advantages}

To the patient, the comprehensive health center offers major advantages. Perhaps the most important of these is improvement in continuity of care. Transfer from one type of health service to another is much easier where such a center exists. The personal physician responsible for the patient's health can follow his progress from one service to another with little difficulty and can readily discuss the care of his patient with the physicians and other health personnel providing specialized services. Another advantage is that the total resources of the health center, including the services of many types of personnel and the full array of equipment, are available to the patient whether he is attending a health department or hospital clinic, is an inpatient, or is in the nursing home unit. Finally, the transportation problem is eased; the patient does not have to travel to a variety of places to obtain the care which he requires.

To the physician, the comprehensive health center provides the opportunity to do more and better work by saving valuable time which would otherwise be spent in the harassing task of shuttling between office and hospital. Hos- pital emergencies would no longer mean that office appointments must be cancelled. More time can be spent with bed patients. Laboratory, X-ray, and other facilities are easily accessible. Consultation with his colleagues is more frequent, and it is easier for him to attend the conferences and other educational opportunities offered by the center. Physicians who would otherwise have little or no contact with hospital practice, and who would therefore run serious risk of falling behind in their professional knowledge and competence, do not face this problem in a comprehensive health center because all physicians in a local community or district would be eligible to practice in the center.

To the health department, the comprehensive health center provides an unparalleled opportunity to work closely with physicians, dentists, and other health personnel in developing preventive and control services. Programs of health education, multiple screening, health maintenance for both children and adults, and other services for the control of acute and chronic disease, including mental illness, are greatly facilitated in such a center. Physicians and other health personnel are more readily available to serve in health department clinics. Referrals to therapeutic services are easier to accomplish. Education of the staff of the center in preventive medicine and community health problems is aided by the participation of health department personnel in staff conferences and other programs of professional education. The personnel of the health department also gains a great deal from such participation by keeping abreast of developments in diagnosis and treatment.

To the community, the comprehensive health center makes it possible to provide more and better health service at lower cost. The combination of facilities at a single site prevents unnecessary and expensive duplication of personnel, services, and equipment. More efficient utilization of hospital beds is made possible because of the easy availability of alternative methods of care. Savings in time which would otherwise be wasted in transportation from one facility to another result in more effective use of health personnel. The eligibility of all 
physicians in the community to practice at the center and the greater opportunities for consultation and education of health personnel contribute to a higher level of health services in the community.

Many of these advantages of the comprehensive health center have been documented in two studies which have considered different aspects of such centers, joint housing of hospitals and health departments (6) and physicians' private offices at hospitals $(\eta, 8)$. The difficulties uncovered by these studies were relatively minor ones or resulted from situations which are not inherent in the comprehensive health center concept.

\section{Difficulties}

In a few instances such centers have been relatively inaccessible to some segments of the population. Location must be considered when planning the construction of comprehensive health centers or the utilization of existing hospitals as the nuclei for the centers. It is axiomatic that a comprehensive health center must be readily available to the population it serves.

Where health departments have been housed in hospitals operated under other auspices, the pressure for more hospital space has caused, in a number of instances, a reduction in the space allotted to the health department. This has not occurred in situations where the health department operated the hospital or occupied a separate building on the same site.

It must be recognized that where the comprehensive health center includes units operated by different agencies, coordination is more difficult to achieve. Effective coordination of services will, in such instances, require regular consultation and planning by the participating agencies. Even where a single agency operates the center, the full integration of preventive, therapeutic, and rehabilitative services will not be attained without careful planning and continued attention.

\section{Dimensions}

The size of a comprehensive health center is determined by a number of factors including (a) the distribution of the population to be served; $(b)$ the size and nature of its disease problems; (c) the availability and speed of transportation; $(d)$ the need, on one hand, to have facilities large enough to permit effective service; and $(e)$ the desirability, on the other hand, of having facilities small enough to permit close professional contacts and the encouragement of warm, humane, and personal treatment of patients by the professional staff.

In general, a center serving approximately 50,000 persons would probably represent the optimal size for a comprehensive health center. If we apply current estimates of needs for hospital beds $(9)$, such a center would have 200 to 250 beds for acute general illness, 100 for chronic disease, 150 for patients needing nursing home care, and 250 for those with mental illness. Also, if we use the present national ratios of 1 physician per 750 persons and 1 dentist per 1,700 persons, such a center would provide offices for approximately 65 physicians and 30 dentists. These estimates are gross approximations based on current practice; they will be modified by local variations, scientific advances, and changing needs and demands for health service.

The center would include health department offices, preventive and treatment clinics, a rehabilitation service, and offices for home care personnel. If the hospital is a public institution, the local or district health officer should be responsible for administration of the entire center; if not, he should be an ex officio member of the board of trustees of the hospital. In the latter instance, it may be preferable to have the health department housed on the hospital grounds rather than within the hospital proper. The private offices for physicians and dentists should be leased at rentals equivalent to those charged in other office buildings in the community $(r, 8)$.

The inclusion of beds for mental hospital patients within the center is consistent with present concepts of the management of mental illness. Many general hospitals now maintain beds for acute mental illness. If the health center is to include beds for long-term patients, there is no sound reason why beds for patients with chronic mental illness should not also be provided. The advantages to such patients are significant; they would have available skilled general medical services which are often diff- 
cult to obtain in separate mental institutions, and they would remain in their own communities in regular contact with their families. Both psychiatric and general medical personnel would benefit from the closer association and more readily available consultation resulting from this arrangement.

It would appear doubtful that a comprehensive health center should serve more than 100,000 persons at one extreme and less than 10,000 persons at the other. In isolated rural areas it may be necessary to build smaller centers, but these should be kept to a minimum because of the difficulties of providing effective services in centers with less than 50 beds for acute illness. The other alternative, to maintain independent health centers without hospital beds, is not a good one; it would be preferable to establish such centers, where necessary, as outposts of the comprehensive center with its personnel manning them.

In addition to the local community or district health centers, the medical schools and other large centers would provide patients in their regions with the more highly skilled, complex, and specialized services. Each medical school center would be responsible not only for consultation, referral, and educational services for the local centers in its region, but would serve as the local center for its immediate neighborhood in order to maintain an adequate service base for the education of health personnel.

\section{Feasibility}

The development of comprehensive health centers is complicated by the wide variety of voluntary and governmental agencies which have responsibilities for the construction and operation of health facilities. A major step facilitating the establishment of such centers would be the amalgamation of the governmental health services in a community-including public health, hospital, and rehabilitation services-in a single health department. Cooperative arrangements can be developed between the health department and the voluntary hospitals in a variety of ways.

For example, in a local community or district with a voluntary hospital but no governmental hospital facility, the health department building can be constructed adjacent to the voluntary hospital. The hospital can provide all necessary hospital and nursing home services for patients whose care is paid for by public funds from the health department, and various types of arrangements can be made for the joint development of preventive clinic services. The wing or building for physicians' and dentists' offices would be operated by the voluntary hospital.

In another district there may be a governmental hospital facility serving needy patients but no facility for those able to pay fully for their care. Here the needs of the community may be met either by expansion of the governmental hospital to provide services for those able to pay or by construction of an adjacent voluntary hospital. The health center would be further developed by the addition of nursing home facilities, health department offices, and facilities for physicians' and dentists' offices. Such arrangements are quite feasible; a number of centers already exist where governmental and voluntary institutions are grouped at a single site.

Where the existing hospital facility is unsuitable to become the nucleus of a comprehensive health center, or where no hospital facility exists, new construction will be necessary. Such situations provide an excellent opportunity to formulate integrated plans for truly comprehensive health centers. There will be a great need for experimentation with different types of plans, and the construction of demonstration health centers can play an important role in helping to guide future developments.

\section{Adaptability}

The comprehensive health center is adaptable to a variety of methods of organizing and financing health services. The concept that health services for local communities or districts should be grouped together in a single, accessible health center is independent of whether the services are paid for by the individual, by prepayment, or by tax funds. Similarly, the physicians and dentists who practice in the center may be in private practice, either as solo or group practitioners, or they may be on a 
part-time or full-time salaried basis. All of the various possibilities may exist side by side in a single center.

A great advantage of the comprehensive health center is that it permits variation, growth, and change. Within each center, it will be possible for practitioners to develop informal or formal group practice arrangements as they see fit to do so and to abandon such arrangements when they prove unsuitable. Similarly, it will be possible in each health center to vary the health services to meet changing needs, to use facilities flexibly, and to establish new services and facilities when necessary.

\section{Conclusion}

The comprehensive health center, which brings together all preventive, therapeutic, and rehabilitative health services of a local community or district at a single site, is recommended as the basic facility for the provision of local health services. The development of such facilities is consistent with both the theoretical orientation of public health and the practical necessities of current health programs and would provide a suitable framework for the further evolution and improvement of local community health services.

\section{REFERENCES}

(1) Terris, M.: Hermann Biggs' contribution to the modern concept of the health center. Bull Hist Med 20 : 387-412, October 1946.

(2) Medical care for the American people: The final report of the committee on the costs of medical care. University of Chicago Press, Chicago, 1932.

(3) Mountin, J. W., Pennell, E. H., and Hoge, V. M. : Health service areas: Requirements for general hospitals and health centers. PHS Bulletin No. 292, U.S. Government Printing Office, Washington, D.C., 1945.

(4) Medical care in a national health program. Amer J Public Health 34: 1252-1256, December 1944.

(5) Coordination of hospitals and health departments: Joint statement of the American Haspital Association and American Public Health Association. Amer J Public Health 38: 700-708, May 1948.

(6) Terris, M.: Joint housing of hospitals, health departments, and laboratories. A survey of joint housing. Amer J Public Health 41: 319-325, March 1951.

(y) Rorem, C. R.: Physicians' private offices at hospitals. Amer J Public Health 48: 874-879, July 1958.

(8) Rorem, C. R.: Physicians' private offices at hospitals. Hospital Council of Philadelphia, 1958.

(9) Abbe, L. M., and Baney, A. M. : The nation's health facilities : ten years of the Hill-Burton hospital and medical facilities program, 1946-1956. PHS Publication No. 616, U.S. Government Printing Office, Washington, D.C., 1958.

\section{Grants for Health Needs of Migrants}

Surgeon General Luther L. Terry of the Public Health Service has announced the first grants under the Migrant Health Program. Totaling $\$ 1,305,690$, these grants were awarded to 35 State and local health departments and private nonprofit organizations in 21 States. They will partially support projects to improve health conditions and health services for domestic agricultural migrants.

The Migrant Health Program was authorized by an act of Congress approved by the President on September 25, 1962. Its primary emphasis is on establishment of family health service clinics scheduled at times and places accessible to migrants and without a residence requirement. However, nursing, sanitation, health education, and other health services for migrants are eligible for support. One project operates a mobile medical clinic staffed by physicians, nurses, and a dentist. In another project public health nurses visit the camps for early detection of disease and referral of patients to the clinics or the local hospital. One project advises camp owners on how to correct deficiencies in facilities and avoid unsanitary conditions in future building.

"The projects represent the beginning of a 3-year program designed to help meet the urgent health needs of our domestic agricultural migratory workers and their families," Dr. Terry said. 